\title{
SURGERY OF DEFORMITIES RESULTING FROM WAR INJURIES OF THE EXTREMITIES.
}

\author{
By T. P. McMURRAY, M.Ch., F.R.C.S. (Ed.) \\ (Professor of Orthopadic Surgery, Liverpool University.)
}

Deformities resulting from war injuries may, with advantage, be classified into two groups, the first comprising those in which deformity is unavoidable on account of the irretrievable loss of tissue, and the second group-which is unfortunately larger than it ought to be-where part at least of the deformity could have been prevented by intelligent treatment or after care.

In the first group, even though some degree of deformity is inevitable, much can be done to minimise subsequent functional disability and prevent the appearance of obvious disablement of which the patient remains peculiarly conscious. The second group is-from the surgeon's point of view-even more important than the first, and the occurrence of preventable disability should be considered as a blot on the efficiency of the hospital and of the surgeon in charge.

Many illustrations of this failure of preventive surgery spring to the mind as, for instance, where a soft tissue wound in the region of a joint has been allowed to heal with the joint fixed at such an angle that all useful movements are lost. Even though the presence of scarring may lead to a diminution of movement it is comparatively simple to prevent its contraction producing a crippling deformity of the limb.

It is impossible in a short paper to deal with all the deformities which may occur as a result of war wounds, and it is, therefore, advisable that we should confine our attention to those commonly seen during, and after, the last war. Every conceivable distortion was encountered sooner or later, but certain disabilities occurred so constantly that they became recognised as major problems. The amount of crippling and disability produced by any deformity does not entirely depend on its severity. Thus, the usefulness of the upper limb may be retained even in the presence of severe deformity caused by mal-union of the long bones, so long as the deformity does not interfere with joint movements. On the other hand, even an apparently slight degree of displacement of a joint may produce a crippling disability. Probably the most typical example of this can be seen in those patients in whom the joints of the hands and fingers have become rigid through neglect, or inefficient splintage.

\section{Rigidity of the Metacarpo-phalangeal Range.}

When a motor nerve has been injured, overstretching of the muscles supplied must be prevented by the application of some apparatus which will hold these muscles in the relaxed position. The importance of this splintage of paralysed muscles was recognised at an early stage in the last war, and after injury to the musculo-spiral nerve - either temporary or permanent-a dorsiflexion hand splint was immediately applied to relax the paralysed extensor muscles. The first type of splint supplied for this purpose consisted of two flat pieces of metal joined together at an angle of about 45 degrees. When the hand was retained on such a splint for months, or even for weeks, hyperextension at the metacarpo-phalangeal and interphalangeal ranges was unavoidable, and after removal of the splint, flexion at these joints was found to be seriously restricted, or even impossible. Usually this rigidity resulfed from a combination of two factors, the first being the continued fixation of the joints in hyperextension, and the second a chronic œdema 
of the tissues, produced by a wound in the forearm, arm or shoulder region. Without hyperextension of the joints any rigidity produced, even by long continued œdema of the tissues was slight, and rapidly responded to treatment. When, however, the two conditions of hyperextension and odema were combined the resulting rigidity was disabling, and in some instances could not be corrected, even after months of physio-therapy.

This rigidity can be prevented by the use of a splint which maintains the wrist in dorsiflexion, and is so moulded in its palmar portion that the metacarpophalangeal and interphalangeal joints remain in a position of about 30 degrees flexion. This position of flexion may be described as a normal position of these particular joints, at which they may be maintained almost indefinitely without risk of permanent disability. The importance of preventing this loss of function in the hand and fingers cannot be overstated, as in the last war most massage and physio-therapy departments spent a considerable proportion of their time trying-with little success-to improve the condition.

\section{TREATMENT.}

If, however, the deformity has been allowed to occur it will be found that, even under anæsthesia, flexion of these joints is impossible. In the movement of flexion at the metacarpo-phalangeal joint the base of the first phalanx glides over the head of its metacarpal, a movement which depends on the mobility of the capsule and the extensor expansion on the back of the joint. When these tissues have become shortened, thickened and fibrotic, the articular margin of the base of the phalanx rests on the apex of the head of the metacarpal, and attempts to flex the joint simply force the anterior margin of the base of the phalanx into the articular surface of the head of the metacarpal.

Treatment then should be directed to flexing the joints, whilst at the same time endeavouring to separate the articular surfaces by distraction. This double effect can be produced by the use of the Banjo splint, consisting of a curved wire ring fixed to a wrist splint or plaster case. Hooks are attached to the wire ring, from which traction on the fingers is provided, and as the ring is gradually bent in a palmar direction flexion occurs at the affected joints. The process is a slow and tedious one, the use of the splint extending usually over a period of 2-3 months, and full restoration of function being problematical.

\section{Mal-union.}

The amount of disability produced by mal-union of a fracture depends on the severity of the mal-alignment, and also on the site of deformity and on the bone affected. Thus, angular deformity of the humerus, or of the radius and ulna, may cause comparatively little disability so long as the fracture is situated in the shaft of the bone and does not interfere seriously with the movements of the limb, which constitute its main function.

An equal amount of deviation in the lower limb, either above or below the knee, causes considerably greater disability. When weight is borne on the malunited limb the strain, which should pass directly from the femur through the tibia and astragalus, is borne partly by the ligaments of the side corresponding to the angle of deformity. Thus, in the presence of outward bowing of the femur, weight is carried partly by the external lateral ligament of the knee joint. If, however, the mal-union of the femur consists in overriding of the fragments, without any 
alteration in the alignment of the limb, weight is borne as before and normal function is retained in the knee and ankle. The ill effects of loss of alignment of the lower limb are not confined to strain on ligaments but, as a result of unequal pressure on the joints, traumatic osteo-arthritis is gradually developed.

With mal-union of the femur outward or posterior bowing occur most commonly, although inward or forward bowing are also seen occasionally. With simple fractures of the femur such mal-alignment can be prevented easily, but in the severely shattered limbs there is occasionally great difficulty in procuring sufficient extension to counteract these tendencies. If the fractured limb is adequately extended for a sufficiently long period, outward, inward or forward bowing cannot occur, and if a firm support is given posteriorly the tendency to sag at the site of fracture is prevented.

Mal-union below the knee usually consists of bowing inwards at the site of fracture. If extension is used in the treatment of a fractured tibia, or if the limb is fixed in plaster, care must be taken to prevent the obliteration of the normal outward curve of the shin. If such mal-union has occurred its main ill-effects are produced as a result of weight bearing. The foot is deviated outwards, body weight is borne largely on its inner margin, producing abnormal strain on the internal lateral ligament of the ankle, and to some extent on the inner ligament of the knee joint.

Backward bowing of a fracture of the thigh or leg also causes a very disabling type of deformity, the knee becomes hyperextended, the posterior ligament at first takes all the strain, but gradually stretches and permits a further hyperextension of the joint with pain, swelling and later the development of an arthritic condition.

\section{TREATMENT.}

Simple rules can be enumerated for the treatment of deformities of the bones of the limbs. In the upper limb if the mal-union is so extreme, or in such position that it interferes with the usefulness of joint movements, an attempt should be made to restore the normal alignment.

In the lower limb the criteria for correction are somewhat different; any malunion, which leads to the development of abnormal strain on the knee and foot, should be corrected if possible before arthritic changes have developed in these joints. Like all other general rules these are open to occasional exceptions-the patient's general health, or the presence of sepsis in the neighbourhood may prevent any operative interference, and efforts should then be made to assist the patient temporarily by suitable supports, wedges or splints.

In the absence of any definite contra-indication the object of operation should be the restoration of the normal alignment of the limb. Before any open operative procedure is undertaken all traces of lurking sepsis must be eliminated. After the extensive infection of the tissues, usually associated with any war wounds, open operations may light up sepsis which had remained quiescent. These tragedies can be avoided; before opening any bony tissue in which sepsis has been present, an interval of at least six months' freedom from all signs of inflammation must be insisted upon. The area is subjected during the latter part of this period to deep massage and radiant heat, with the object of bringing to light any latent organisms. When it is decided that all sepsis has been eliminated the operation may be undertaken; in some cases the bone may be refractured along the original line, but, as a rule, it is quite sufficient, and much safer, to correct the deformity by means of a simple osteotomy performed near, but not through, the mass of 
new formed callus. After the osteotomy treatment is carried out as for a recent simple fracture of that region. The difficulties are less than in the original wound, sepsis is absent, the patient is not usually ill, and a successful correction is to be expected.

\section{Non-union.}

Non-union of a compound fracture, either in civilian or in military surgery, is in some cases unavoidable, but in many instances it can be prevented by intelligent treatment. In a compound wound, especially in one caused by a bullet or a piece of shell, it is naturally essential that the débridement of the whole wound should be extensive and, as far as possible, complete. Loose pieces of bone should be removed, even when the detached fragments are large, but when these broken pieces of bone are still attached to muscle and have presumably a living blood supply, their removal from the wound should be avoided. Unnecessary removal of bone may undoubtedly produce non-union, but this error of surgical technique is by no means the sole cause of failure of union. During the last war fractures of the humerus were frequently treated by continuous traction in an effort to preserve alignment of the bone, and to prevent overriding. If the traction was sufficiently powerful these two desiderata of correct alignment and full length were obtained, but in many instances a fracture so treated failed to unite.

In many bones-such as the tibia, fibula, or femur-a certain amount of overextension does not prevent union but, in the case of a fractured humerus, full length of the limb can be obtained easily by using the force of gravity alone, and any traction must be very carefully controlled in order to avoid this complication. In fact, in a compound fracture of the humerus a small amount of overlapping of the bone tends towards union of the fracture, and the consequent shortening of the arm leads to little or no disability.

The degree of disablement caused by non-union depends to a large extent on the bone affected, and to a certain degree also on the site at which the nonunion has occurred; thus non-union of a femur is a crippling disability, whilst a similar condition of the ulna may lead to comparatively slight loss of function. Non-union can on occasion be treated successfully by efficient fixation of the fracture, and this statement is of importance because many surgeons fail to recognise the difference between delayed and non-union. It is impossible to put a time limit on the process of union; in some cases with adequate treatment and without any apparent cause, consolidation of a fracture may not be complete for a period of $3-6$ months. It is, therefore, essential to make certain by clinical and X-ray examinations that the condition present is in reality a complete non-union, and not simply an unusually slow, but otherwise normal process of consolidation, before proceeding to operation.

\section{Ankylosis.}

When it becomes apparen $\ddot{t}$ that ankylosis is inevitable, the limb should be supported so that it may occur at the angle which is the most useful from the point of view of the subsequent function of the limb, which position naturally varies with the joint affected.

Wrist. In the wrist joint loss of movement causes the minimum of disability when the hand is fixed at an angle of $15-20$ degrees dorsiflexion to the line of the forearm. Extreme dorsiflexion is to be avoided as it prevents the patient from 
engaging in many of the more delicate forms of manual labour. The degree of disability is even greater when the joint is fixed in palmar flexion, as the strength of the grip diminishes directly in proportion to the amount of flexion, until in full flexion it is almost entirely lost.

Elbow. Ankylosis of this joint at a position of I5 degrees below a right angle causes the least inconvenience to the general usefulness of the limb. It is true with an elbow ankylosed in this position the patient cannot use a fork in the normal way-or in the case of a woman cannot brush her hair-but for all ordinary occupations this angle of fusion has been found to be the most satisfactory.

Shoulder. Ankylosis of the shoulder joint at any position produces a very definite disability and causes great inconvenience to the patient but, as in the wrist and elbow, the amount of disability can be minimised if the joint is fixed at an angle of $45-60$ degrees abduction. If the ankylosis occurs with the arm at an angle of greater abduction complete adduction is difficult, or even impossible and the limb is subject to constant injury, whilst in addition the patient often complains of aching and tiredness round the shoulder girdle.

Hip. Ankylosis of the hip causes great inconvenience to the patient in whatever position the joint is fixed, but the disturbance is least when the fusion has taken place with the limb in the neutral position, and at an angle of 20 degrees flexion. If the limb is abducted the consequent pelvic tilting disturbs the mechanism of the other hip, and causes a feeling of strain in the lumbo-sacral region. In fact, a position of adduction at the ankylosed hip causes less disability than that of abduction. The only exception to this rule occurs in those patients in whom there has been considerable loss of length in the limb, when not more than I5 degrees abduction at the ankylosed joint is of advantage.

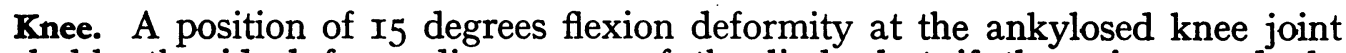
is probably the ideal for ordinary use of the limb, but if there is any doubt regarding the solidity of the fusion the straight position of the limb is to be preferred. The reason for this preference can be appreciated easily; when the ankylosis is unsound, weight bearing tends to alter the angle of fusion when the knee is bent, while in the straight position this tendency does not occur.

Ankle. The right-angled position with the foot in slight valgus deformity constitutes the best functional position, and interferes only to a very slight dégree with the normal functions of the limb.

\section{Types of Ankylosis.}

Clinically ankylosis may be divided into two types according to the reaction of the fused joint to the resumption of function of the affected limb.

1. Sound ankylosis, in which the tissue joining the bone ends is either bony or fibrous in structure, but in neither case so firm that the position of the joint does not alter with the resumption of function.

2. Unsound, in which the fusing material is always fibrous and of such a nature that alteration of position of the limb occurs when the joint is subjected to strain.

Correction of Ankylosis. If it is desired to alter the position of ankylosis the necessary procedure depends on the character of the fusion. In all cases of sound ankylosis correction is only possible by open operation. In most cases of 
unsound ankylosis manipulative correction of the angle of fixation is comparatively simple, but its very simplicity indicates that a re-development of the deformity is likely to occur unless the joint is fixed in its new position, either by operation or by long continued splintage. In most instances joints which have become ankylosed as a result of war wounds are not suited for such operative procedures as arthroplasty. The soft tissues have been so widely destroyed and infected that, even if a remodelling of the bone ends could be procured, restoration of voluntary movements in the joint is usually an impossibility. If the joint has been the seat of severe infection any open operative procedure should be delayed until at least 4-6 months following the cessation of discharge. This interval, which is similar to that employed before operation for correction of mal-union, usually prevents the tragedy of a flare up, and allows healing to take place without any complications. 